\title{
miR-133b is a potential diagnostic biomarker for Alzheimer's disease and has a neuroprotective role
}

\author{
QIN YANG $^{1^{*}}$, QIULING ZHAO ${ }^{2^{*}}$ and YANLIANG YIN ${ }^{3}$ \\ ${ }^{1}$ Department of Neurology; ${ }^{2}$ Digestive Endoscopy Center and ${ }^{3}$ Department of Health Care, \\ Dongying People's Hospital, Dongcheng, Shandong 257091, P.R. China
}

Received January 25, 2019; Accepted July 12, 2019

DOI: $10.3892 /$ etm.2019.7855

\begin{abstract}
MicroRNAs (miRNAs/miRs) are involved in post-transcriptional gene regulation and aberrant expression of miRNAs has been widely detected in various human diseases. The aim of the present study was to examine the serum levels of miR-133b in patients with Alzheimer's disease (AD), and to explore its diagnostic value and neuroprotective role in AD. Reverse transcription-quantitative PCR was applied to analyze the serum levels of miR-133b in 105 AD patients and 98 healthy controls. A cell model of AD was established by treating SH-SY5Y cells with amyloid $\beta(\mathrm{A} \beta) 25-35$, and the resulting effect on miR-133b expression was determined. Cell viability and apoptosis were also measured. A dual-luciferase assay was used to validate a target gene of miR-133b. Receiver operating characteristic (ROC) curve analysis was also applied to assess the specificity and sensitivity of miR-133b to diagnose AD. The results indicated that the serum levels of miR-133b were significantly downregulated in AD patients and SH-SY5Y cells treated with A $\beta 25-35$ (all $\mathrm{P}<0.001$ ). A positive correlation between the serum levels of miR-133b and the Mini-Mental State Examination score of AD patients was determined ( $\mathrm{r}=0.8814, \mathrm{P}<0.001)$. The area under the ROC curve for miR-133b regarding the diagnosis of AD was 0.907 , with a sensitivity of $90.8 \%$ and specificity of $74.3 \%$ at the cutoff value of 1.70 . Overexpression of miR-133b significantly attenuated the $A \beta 25-35$-induced inhibition of cell viability $(\mathrm{P}<0.01)$ and induction of cell apoptosis $(\mathrm{P}<0.01)$. The luciferase reporter assay demonstrated that epidermal growth factor receptor (EGFR) is a target gene of miR-133b. In conclusion, miR-133b may serve as a novel diagnostic biomarker for AD and it may have a neuroprotective role in AD and targets EGFR.
\end{abstract}

Correspondence to: Professor Yanliang Yin, Department of Health Care, Dongying People's Hospital, 317 South 1st Road, Dongcheng, Shandong 257091, P.R. China

E-mail: liyan992592@163.com

\section{${ }^{*}$ Contributed equally}

Key words: microRNA-133b, diagnosis, Alzheimer's disease, epidermal growth factor receptor

\section{Introduction}

Alzheimer's disease (AD) is a chronic age-associated neurodegenerative disorder and the leading cause of dementia in elderly individuals $(1,2)$. AD is characterized by cognitive dysfunction and memory loss (3). It is estimated that the global prevalence of dementia has reached as high as 24 million, which may double every 20 years, i.e. by 2040 (4). The pathogenic mechanisms of AD include the increase of inflammation, death of neurons and atrophy of the brain (5). The clinical pathology of AD is aberrant amyloid protein deposition of amyloid $\beta(A \beta) 42$, as a result of abnormal amyloid precursor protein processing. To date, the etiological mechanisms underlying the neuropathological changes of AD remain to be fully elucidated and diagnostic biomarkers for the early detection of AD remain limited (6). Thus, it is of great significance to identify more AD-associated genes and further develop screening methods for these genes to improve the diagnosis and therapy for AD.

MicroRNAs (miRNAs/miRs) are a class of small non-coding RNAs with a length of 20-24 nucleotides (7). miRNAs regulate the degradation or translational repression of their target mRNAs through binding to their 3'-untranslated region (UTR) (8). Dysregulation of miRNA has been reported to participate in multiple biological and pathological processes, including AD. For instance, Kumar et al (9) reported that miR-455-3p was upregulated in AD patients compared with that in healthy controls, suggesting the potential role of miR-455-3p in the pathogenesis of AD. Hong et al (10) indicated that miR-125b, miR-9, miR-191-5p and miR-28-3p may be potential biomarkers for AD, and their expression levels were validated in SH-SY5Y cells and in a mouse model.

miR-133b is regarded as a specific miRNA of the muscle, and it may be involved in myoblast differentiation and certain myogenic diseases (11). Of note, miR-133b has been indicated to have the ability to inhibit the maturation and function of midbrain dopaminergic neurons in primary rat midbrain cultures (12). AD and Parkinson's disease (PD) all belong to neurodegenerative diseases, which are characterized by neurodegenerative changes and brain dysfunction (13). Furthermore, multiple studies have proved the abnormal expression of miR-133b in PD patients, indicating the crucial role of miR-133b in this neurodegenerative disease (14-16). However, to date, the expression and role of miR-133b in the development of AD have remained to be fully determined. 
Epidermal growth factor receptor (EGFR), also named as ErbB-1 or HER1 in humans, belongs to the ErbB family. It is a significant transmembrane receptor and has been reported to have tyrosine kinase activity (17). EGFR may be involved in various cellular processes, including cell survival, differentiation, proliferation, migration and repair of cell damage $(18,19)$. Furthermore, the key ligands of the EGFR protein, EGFs, has been indicated to regulate brain development, and control the survival and function of nerve cells, suggesting a potential role of EGFR in the development of AD (20). Furthermore, in esophageal squamous cell carcinoma (ESCC), miR-133b was proved to inhibit cell cycle progression and bioinformatics analysis indicated that there was a binding site of miR-133b on the 3'-UTR of EGFR (21). Thus, it may be hypothesized that EGFR is a target gene of miR-133b in AD.

In the present study, the expression and diagnostic value of miR-133b in patients with AD were assessed, and its neuroprotective role in this disease was further explored.

\section{Materials and methods}

Study population. The protocol of the present study was reviewed and approved by the Ethics Committee of Dongying People's Hospital (Dongcheng, China). Written informed consent was obtained from each participant.

A total of 105 patients with $\mathrm{AD}$ were recruited for the present study, who were diagnosed according to the National Institute on Aging-Alzheimer's Association criteria (1). All patients were aged between 60 and 85 years, and were admitted to Dongying People's Hospital between July 2014 and January 2017 (Dongcheng, China). Patients fulfilling the following criteria were excluded from the study: i) Patients receiving heparin therapy at the time of the blood collection, as heparin may interfere with RNA isolation; ii) patients who had other diseases/conditions, including acute myocardial infarction, cancer, PD and multiple sclerosis (since these diseases may give rise to expression differences of miRNA) (22). A total of 98 age- and gender-matched healthy individuals were randomly selected as a control group when receiving a routine physical examination. Each enrolled healthy control had normal neurological function. The neurological function of the controls was determined according to their clinical features, laboratory examination results and Mini-Mental State Examination (MMSE) scores. High blood pressure (HBP), diabetes mellitus (DM), coronary atherosclerotic heart disease (CAHD) and hyperlipidaemia (HLP) were diagnosed according to the current diagnostic criteria (23-26). The MMSE score was determined for each participant to assess cognitive and functional impairment (27). As presented in Table I, the AD group had a mean MMSE score of $20.48 \pm 5.42$, while the control group had a higher mean MMSE score of $29.00 \pm 0.64$.

Sample collection. A total of $5 \mathrm{ml}$ peripheral blood was collected from each patient after fasting for $12 \mathrm{~h}$. The serum was immediately centrifuged at $3,000 \mathrm{x}$ g for $10 \mathrm{~min}$ at room temperature, followed by centrifugation at $12,000 \mathrm{x} \mathrm{g}$ for $5 \mathrm{~min}$ at $4^{\circ} \mathrm{C}$. The separated plasma was stored at $-80^{\circ} \mathrm{C}$ until analysis. The serum samples were examined by measurement of miR-133b expression two weeks from the time-point of sample collection and the data were collected.
Cell culture and transfection. The human neuroblastoma cell line SH-SY5Y was purchased from the American Type Culture Collection. It was maintained in high-glucose Dulbecco's modified Eagle's medium (Invitrogen; Thermo Fisher Scientific, Inc.) containing 15\% fetal bovine serum (Invitrogen; Thermo Fisher Scientific, Inc.) and penicillin/streptomycin $(100 \mu \mathrm{g} / \mathrm{ml}$; Keygen Biotechnology). The cells were cultured in a humidified incubator with $5 \% \mathrm{CO}_{2}$ at $37^{\circ} \mathrm{C}$. The medium was changed every day during cell growth. When the cells reached $80-90 \%$ confluence, the cell suspension was sub-cultured on a scale of $1: 3$. For the $A \beta$ group, cells were seeded into a 96 -well plate at a density of $1 \times 10^{4}$ cells per well, and after culture for $24 \mathrm{~h}$, medium supplemented with A $\beta 25-35$ ( $40 \mu \mathrm{M} / 1$; Sigma-Aldrich; Merck KGaA) was added and the cells were cultured for another $48 \mathrm{~h}$ (28). Prior to use, A $\beta 25-35$ was dissolved in sterile physiological saline and incubated at $37^{\circ} \mathrm{C}$ for one week to allow for fibril formation. Medium containing an equal volume of normal saline was used for the negative control. Each group was independently repeated five times.

The miR-133b mimics, miR-133b inhibitor and the corresponding negative controls of mimics and inhibitor (mimics $\mathrm{NC}$ and inhibitor NC) were provided GenePharma. The sequences were as follows: miR-133b mimics, 5'-UUUGGU CCCCUUCAACCAGCUA-3'; miR-133b inhibitor, 5'-UAG CUGGUUGAAGGGGACCAAA-3'; mimics NC, 5'-UGU AGGGCCACUCAGUCA ACUU-3'; inhibitor NC, 5'-AAU AUGGGCGAAAUGGGGCCAUC-3'. For the different transfection groups, SH-SY5Y cells were seeded into a 96-well plate at the density of $1 \times 10^{4}$ cells per well. After culture for $24 \mathrm{~h}$, cells were first transfected with $100 \mathrm{nM}$ miR-133b mimics, miR-133b inhibitor or corresponding mimics NC or inhibitor $\mathrm{NC}$ at room temperature and then treated with $\mathrm{A} \beta 25-35$ for $48 \mathrm{~h}$. The transfection was performed using X-treme GENEHPDNA transfection reagent (Roche) according to the manufacturer's protocol. At $48 \mathrm{~h}$ post-transfection, the cells were harvested for the subsequent experiments. The overexpression/knockdown efficiency was determined by reverse transcription-quantitative (RT-q)PCR.

$R N A$ extraction and $R T-q P C R$. The total RNA was extracted from the serum of all enrolled participants using the miRVana miRNA Isolation Kit (Ambion; Thermo Fisher Scientific, Inc.), while RNA was isolated from SH-SY5Y cells of the A $325-35$ group and control groups using TRIzol Reagent (Invitrogen; Thermo Fisher Scientific, Inc.) based on the manufacturer's protocol. RT reactions were performed using the miScript Reverse Transcription Kit (Qiagen) with the following reaction conditions: Incubation at $42^{\circ} \mathrm{C}$ for $60 \mathrm{~min}$ and at $85^{\circ} \mathrm{C}$ for $5 \mathrm{~min}$. Real-time PCR was performed with the SYBR green I Master Mix kit (Invitrogen; Thermo Fisher Scientific, Inc.) using the 7300 Real-Time PCR System (Applied Biosystems; Thermo Fisher Scientific, Inc.) with the following reaction conditions: Denaturation at $95^{\circ} \mathrm{C}$ for $3 \mathrm{~min}$ and $45 \mathrm{PCR}$ cycles $\left(95^{\circ} \mathrm{C}\right.$ for $15 \mathrm{sec}, 60^{\circ} \mathrm{C}$ for $20 \mathrm{sec}, 72^{\circ} \mathrm{C}$ for $20 \mathrm{sec}$ and $78^{\circ} \mathrm{C}$ for $20 \mathrm{sec}$ ). The $2^{-\Delta \Delta \mathrm{Cq}}$ method was used to determine the relative expression of miR-133b and data were normalized to U6 (29). The primer sequences were as follows: miR-133b forward, 5'-GCG CTTTGGTCCCCTTC-3' and reverse, 5'-CAGTGCAGGGTC CGAGGT-3'; U6 forward, 5'-CTCGCTTCGGCAGCACA-3' and reverse, 5'-AACGCTTCACGAATTTGCGT-3'. 
Table I. Demographic and clinical characteristics of the control and AD group.

\begin{tabular}{lccr}
\hline Variable & $\begin{array}{c}\text { Control group } \\
(\mathrm{n}=98)\end{array}$ & $\begin{array}{c}\text { AD group } \\
(\mathrm{n}=105)\end{array}$ & P-value \\
\hline Age (years) & $75.38 \pm 7.11$ & $76.46 \pm 5.87$ & 0.242 \\
Gender (F/M) & $44 / 54$ & $45 / 60$ & 0.770 \\
MMSE scores & $29.00 \pm 0.64$ & $20.48 \pm 5.42$ & $<0.001$ \\
Other diagnoses & & & \\
HBP (yes/no) & $42 / 56$ & $49 / 56$ & 0.585 \\
DM (yes/no) & $48 / 50$ & $52 / 53$ & 0.938 \\
CAHD (yes/no) & $46 / 52$ & $49 / 56$ & 0.969 \\
HLP (yes/no) & $38 / 60$ & $45 / 60$ & 0.554 \\
\hline
\end{tabular}

HBP, high blood pressure; DM, diabetes mellitus; CAHD, coronary atherosclerotic heart disease; HLP, hyperlipidaemia; MMSE, mini-mental state examination; F, female; M, male; AD, Alzheimer's disease.

MTT assay. The cell viability was measured using an MTT assay. The stably transfected SH-SY5Y cells were seeded into a 96-well plate at a density of $5 \times 10^{4}$ cells per well and cultured for $24 \mathrm{~h}$. The cells were then incubated with $20 \mu \mathrm{l}$ MTT stock solution (0.5 mg/ml; Sigma-Aldrich; Merck KGaA) and the supernatant was removed after $4 \mathrm{~h}$ of further incubation at $37^{\circ} \mathrm{C}$. Subsequently, the precipitated formazan crystals were dissolved in a solution containing $50 \%$ dimethyl sulfoxide (DMSO) (Sigma-Aldrich; Merck KGaA). After $20 \mathrm{~min}$, the absorbance was assessed at $490 \mathrm{~nm}$ using a microplate reader (ELx800; Bio-Tek Instruments). The relative cell viability was determined as the percentage of the control group.

Apoptosis assay. An Annexin V-FITC Apoptosis Detection kit (Keygen Biotechnology) was used for the assessment of cell apoptosis. Cells of each group were collected and gently treated with EDTA-free trypsin. Subsequently, all of the cells were centrifuged at $270 \mathrm{x}$ g for $5 \mathrm{~min}$ and washed twice with PBS. Subsequently, the cells were re-suspended with the binding buffer and mixed with $5 \mu \mathrm{l}$ Annexin V-FITC and PI staining solution. After incubation at $2-8^{\circ} \mathrm{C}$ in the dark for $10 \mathrm{~min}$, the apoptotic rates were measured using a FACSCalibur flow cytometer (BD Biosciences). CellQuest Pro 3.3 software (BD Biosciences) was applied for data analysis. The apoptotic rates were determined as the sum of early and late apoptotic rates, and experiments for each group were repeated in three independent repetitions.

Luciferase reporter assay. The TargetScan analysis (version 7.1; www.targetscan.org) of the 3'-UTR of EGFR revealed a putative binding site for miR-133b. To identify whether EGFR is a target gene of miR-133b, the luciferase reporter gene assay was performed. The 3'-UTR of the EGFR gene was amplified from human genomic DNA using PCR and a mutation of the seed region in the 3'-UTR of EGFR mRNA was introduced using site-directed mutagenesis with the megaprimer PCR method. The 3'-UTR of EGFR, the mutated PCR fragment and the PmiR-RB-REPORT ${ }^{\mathrm{TM}}$ luciferase vector containing the above fragment, were supplied by a service provider (Guangzhou RiboBio Co. Ltd.). The dual-luciferase reporter assay was performed as follows: Prior to transfection, SH-SY5Y cells were seeded into a 96-well plate at a density of $1 \times 10^{4}$ cells per well and cultured for $24 \mathrm{~h}$. Subsequently, SH-SY5Y cells were co-transfected with $50 \mathrm{ng}$ of either wild-type or mutant-type reporter vector and $200 \mathrm{ng}$ miR-133b mimics or inhibitors and mimic NCs or inhibitor NCs using Lipofectamine 2000 (Invitrogen; Thermo Fisher Scientific, Inc.). At $48 \mathrm{~h}$ post-transfection, the cells were harvested and significant relative luciferase activity was measured using Dual-Luciferase Reporter System (Promega Corp.) according to the manufacturer's protocol. The luciferase activity was normalized to that of the Renilla luciferase internal control.

Statistical analysis. SPSS version 18.0 software (SPSS Inc.) and GraphPad Prism 5.0 software (GraphPad Software, Inc.) was applied for data analysis. Demographic and clinical data were analyzed using an independent-samples t-test or a chi-squared test. Differences between two groups were analyzed by Student's t-test or a one-way analysis of variance, followed by a Tukey's multiple-comparisons test. The correlation between the MMSE score and miR-133b levels was assessed by determining Spearman's correlation coefficient, as the miR-133b levels in the patient and control groups did not follow a normal distribution. Receiver operating characteristic (ROC) curve analysis was applied to determine the specificity and sensitivity of the miR-133b levels regarding the diagnosis of AD. Values are expressed as the mean \pm standard deviation. Each experiment had at least three repetitions. $\mathrm{P}<0.05$ was considered to indicate statistical significance.

\section{Results}

Demographic and clinicopathological characteristics of the study population. A total of 105 patients with AD were enrolled in the present study (median age, 76.46 \pm 5.87 years; 45 females, 60 males). A total of 98 healthy controls were also recruited (median age, $75.38 \pm 7.11$ years; 44 females and 54 males). The demographic and clinicopathological characteristics of the cohort are provided in Table I. The AD and control groups were matched regarding age and gender $(\mathrm{P}>0.05)$. The AD patients had significantly lower MMSE score than the controls $(\mathrm{P}<0.05)$, while the other parameters were not significantly different between the AD group and the control group, including HBP, DM, CAHD and HLP (P>0.05).

miR-133b expression levels in AD patients and $S H-S Y 5 Y$ cells treated with A $\beta 25-35$. The expression levels of miR-133b were determined using RT-qPCR. As presented in Fig. 1A, the serum levels of miR-133b in the AD group were significantly lower than those in the control group $(\mathrm{P}<0.001)$. The miR-133b expression was also assessed in human SH-SY5Y cells. It was noted that miR-133b was significantly downregulated in the A $\beta 25-35$ group compared with that in the control group $(\mathrm{P}<0.001$; Fig. 1B)

Correlation between the level of $m i R-133 b$ and MMSE score. MMSE is a common tool for dementia screening, as it is able to fully, accurately and rapidly reflect the mental status and 
A

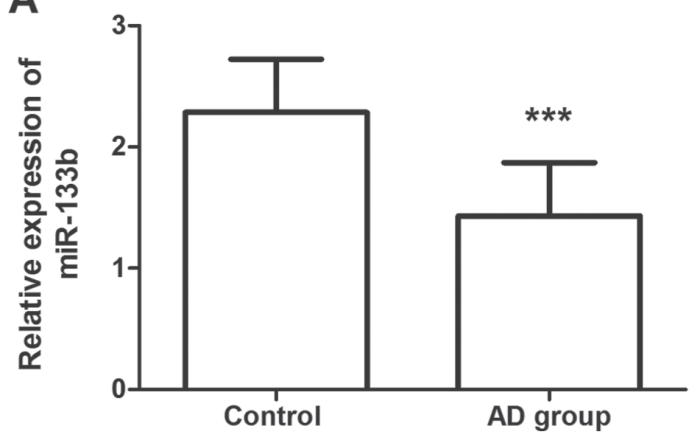

B

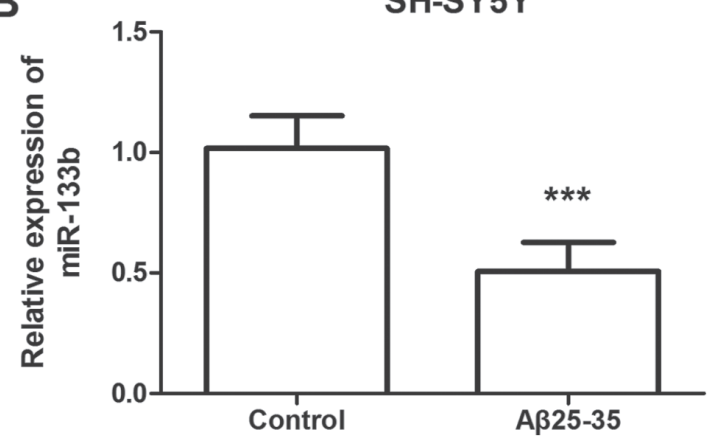

Figure 1. Expression of miR-133b measured by reverse transcription-quantitative PCR in the serum of AD patients and the SH-SY5Y cell line. (A) 105 AD patients and 98 healthy controls were recruited as the AD and control group, respectively. The serum levels of miR-133b were decreased in AD patients compared with those in the healthy controls. (B) The expression levels of miR-133b were significantly downregulated in the A $325-35$-treated SH-SY5Y cells compared with those in the control group. Values are expressed as the mean \pm standard deviation $(\mathrm{n}=5)$. ${ }^{* * *} \mathrm{P}<0.001 \mathrm{vs}$. control. AD, Alzheimer's disease; miR, microRNA; $\mathrm{A} \beta$, amyloid $\beta$.

the degree of cognitive impairment. To examine the association between the serum level of miR-133b and the severity of $\mathrm{AD}$, the correlation between miR-133b expression levels and the MMSE score in AD patients was determined. A positive correlation between the level of miR-133b and MMSE score was identified ( $r=0.8814, \mathrm{P}<0.001$; Fig. 2).

ROC analysis of the diagnostic value of serum miR-133b for $A D$. The ROC curve is a graphical representation reflecting the correlation between sensitivity and specificity of a laboratory test. In the present study, a ROC curve was generated to assess the diagnostic value of serum miR-133b for AD (Fig. 3). The area under the curve for miR-133b was 0.907 , with a sensitivity of $90.8 \%$ and specificity of $74.3 \%$ at the cutoff value of 1.70 . The results suggested that miR-133b may be a sensitive biomarker for differentiating AD patients from healthy individuals.

Effects of miR-133b on A $325-35$-induced neurotoxicity in SH-SY5Y cells. The SH-SY5Y cells were first transfected with miR-133b mimics, miR-133b inhibitor, mimics NC or inhibitor NC. To explore the functional role of miR-133b in $\mathrm{AD}$, an in vitro model of $\mathrm{AD}$ was established by treating the transfected SH-SY5Y cells with A $325-35$ (Fig. 4). The overexpression/knockdown efficiency was determined by RT-qPCR, and the results demonstrated that transfection of miR-133b mimics led to a marked increase in the expression levels of miR-133b, while transfection of miR-133b inhibitor resulted in a significant decrease in its expression, compared with the corresponding negative controls (all $\mathrm{P}<0.001$; Fig. 4A). It was noted that compared with the control group, the viability of A 325 -35-treated SH-SY5Y cells was significantly reduced $(\mathrm{P}<0.001$; Fig. 4B), while cell apoptosis was significantly increased $(\mathrm{P}<0.001$; Fig. $4 \mathrm{C})$. As presented in Fig. 4B, transfection with miR-133b mimics significantly attenuated the $A \beta 25-35$-induced reduction in cell viability, while transfection with miR-133b inhibitor markedly promoted it to cause a further reduction in cell viability (all $\mathrm{P}<0.01$ ). In addition, transfection with miR-133b mimics attenuated the A 325 -35-induced cell apoptosis, while transfection with miR-133b inhibitor further promoted the A $\beta 25-35$-induced cell apoptosis (all $\mathrm{P}<0.01$; Fig. 4C).

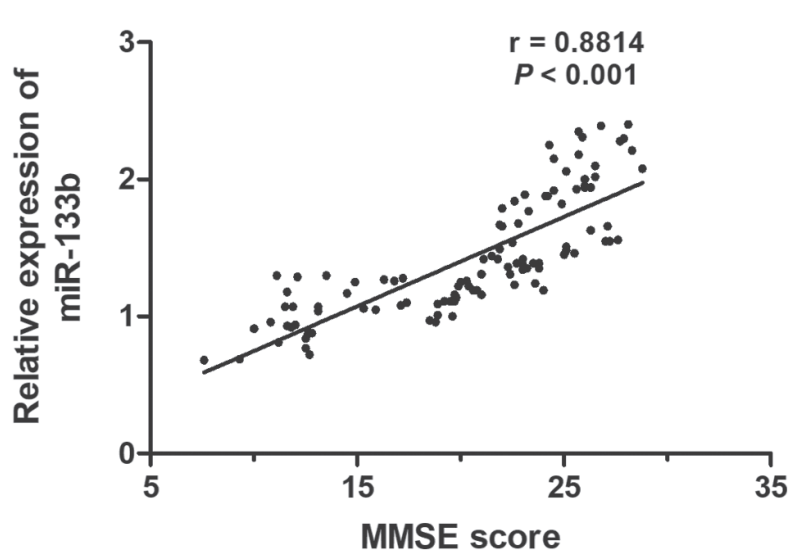

Figure 2. Correlation analysis for the levels of miR-133b and MMSE score in patients with Alzheimer's disease. A positive correlation between the levels of miR-133b and MMSE score was identified. MMSE, Mini-Mental State Examination; miR, microRNA.

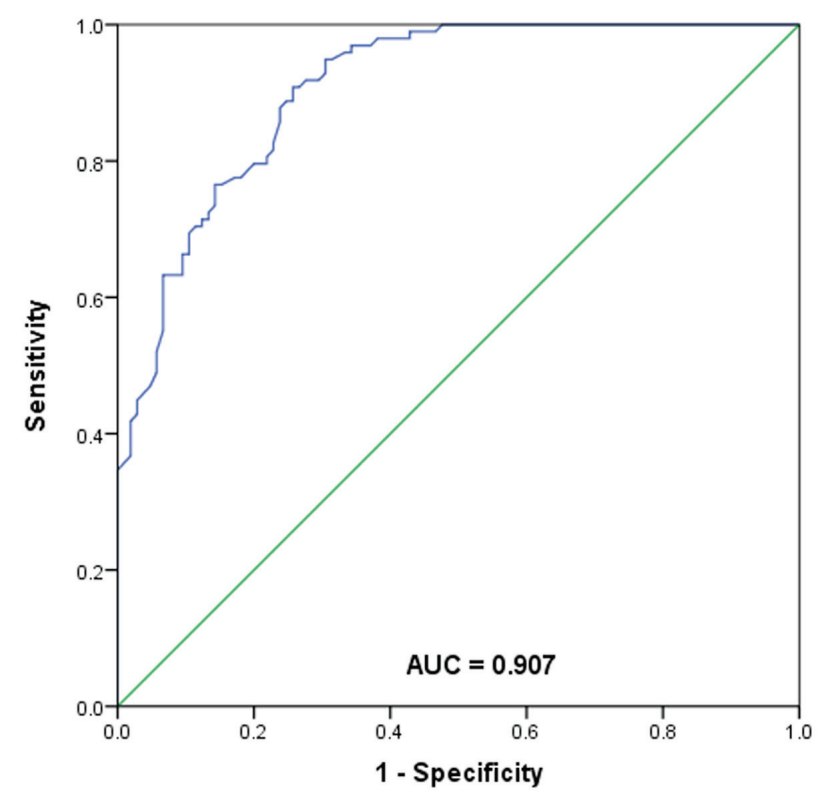

Figure 3. Diagnostic value of microRNA-133b for Alzheimer's disease assessed via ROC curve analysis. The AUC was 0.907 , the sensitivity was $90.8 \%$ and the specificity was $74.3 \%$ (cutoff value, 1.70). ROC, receiver operating characteristic; AUC, area under ROC curve. 
A

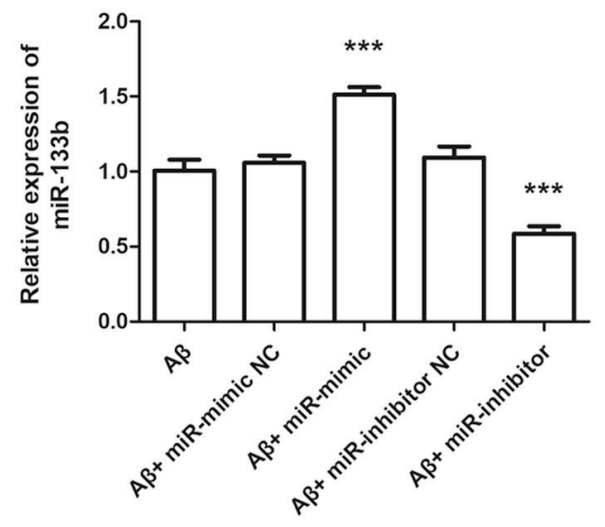

B

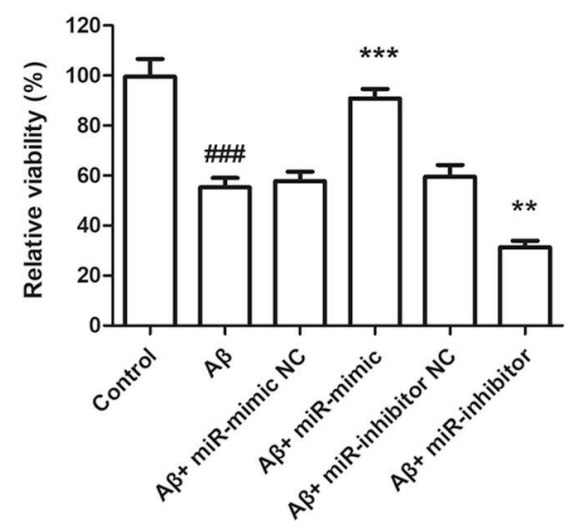

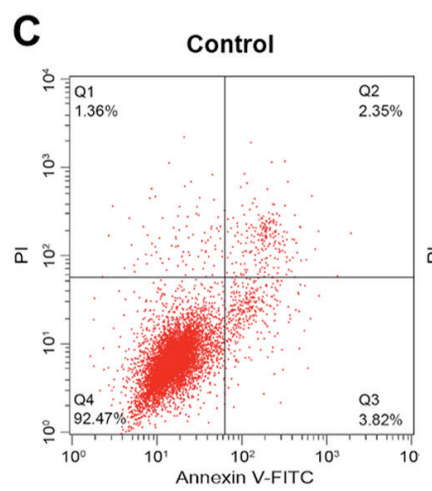
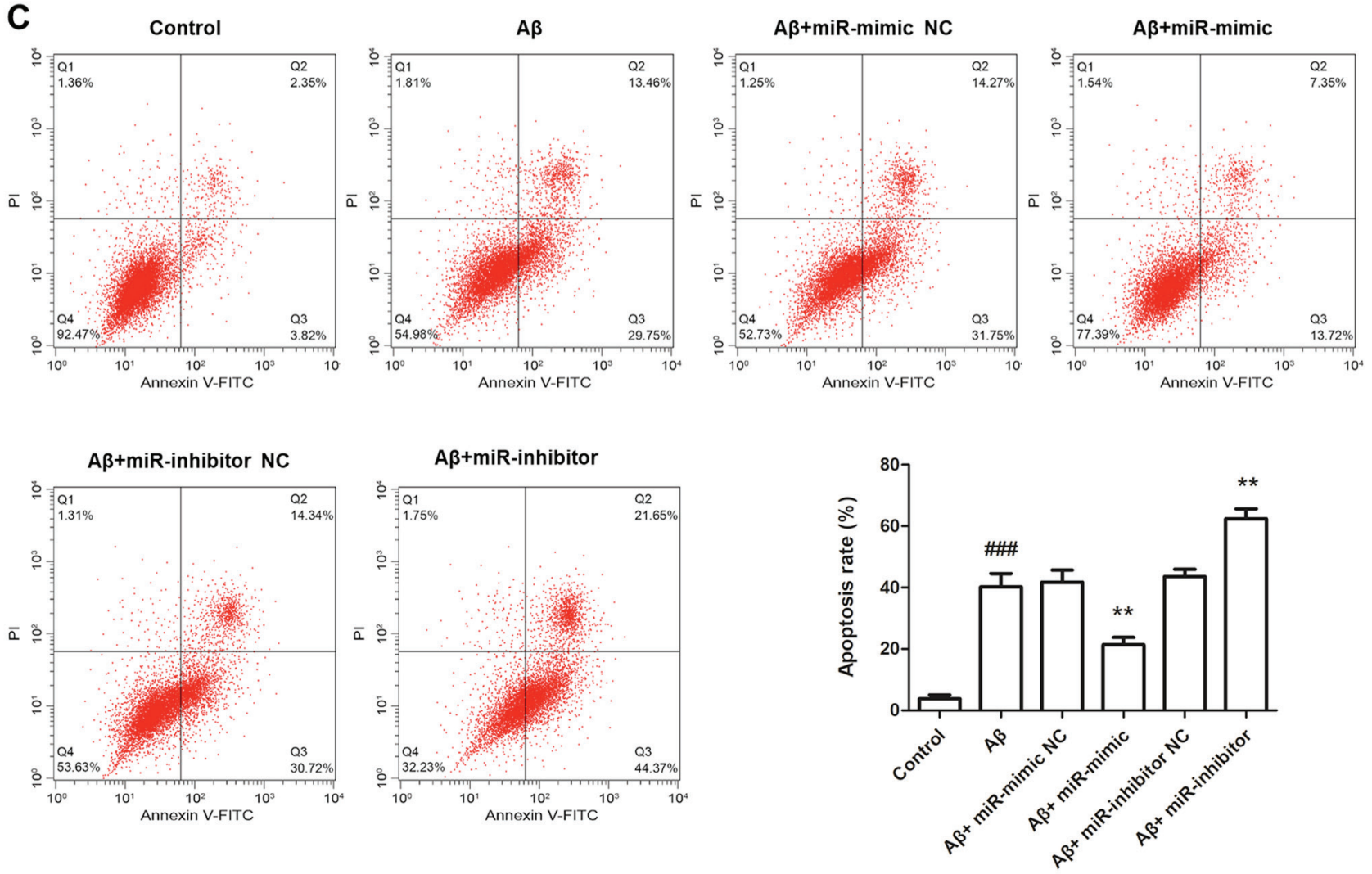

Figure 4. Effects of miR-133b on Aß25-35-induced neurotoxicity in SH-SY5Y cells. (A) In SH-SY5Y cells, the expression of miR-133b was significantly increased by transfection with miR-133b mimics, but was decreased by miR-133b inhibitor compared with that in the corresponding negative controls. (B) Cell viability of SH-SY5Y cells detected via MTT assay. (C) Flow cytometric analysis was performed to detect the apoptotic rate of SH-SY5Y cells. Values are expressed as the mean \pm standard deviation $(\mathrm{n}=3)$. ${ }^{* *} \mathrm{P}<0.01,{ }^{* * *} \mathrm{P}<0.001$ vs. $\mathrm{A} \beta$ group; ${ }^{\# \# \#} \mathrm{P}<0.001$ vs. control. A $\beta$ group, SH-SY5Y cells treated with $\mathrm{A} \beta 25-35$. miR, microRNA; FITC, fluorescein isothiocyanate; PI, propidium iodide; Q, quadrant; NC, negative control; A $\beta$, amyloid $\beta$.

miR-133b directly targets the conserved EGFR 3'-UTR sequence to regulate EGFR expression. TargetScan was utilized to identify target genes of miR-133b, revealing that position 50-56 in the $3^{\prime}$-UTR of EGFR mRNA contained a seven-nucleotide seed match with miR-133b (Fig. 5A). A luciferase reporter assay was applied to confirm the predicted targeting interaction between miR-133b and the 3'-UTR of EGFR. The results suggested that co-transfection with miR-133b mimics attenuated the luciferase activity of the vector driven by the wild-type 3'-UTR of EGFR, while this effect was absent when the luciferase vector driven by the mutant EGFR 3'-UTR was used (Fig. 5B). Conversely, inhibitor of miR-133b promoted the luciferase activity of the vector containing the wild-type 3'-UTR of EGFR, while the luciferase activity of the vector driven by the mutant 3'-UTR of EGFR was not affected (Fig. 5C).

\section{Discussion}

AD is considered to be the principal cognitive disorder in humans. As an effect of the aging of the population, the morbidity of AD has significantly increased in recent years. As there is currently no known cure for AD and limited early diagnostic biomarkers, it is of great importance to explore novel strategies of early diagnosis and treatment for AD. In contrast to cerebrospinal fluid collection, serum collection is 
A hsa-miR-133b

$$
\begin{aligned}
& \text { WT-EGFR 3' UTR } \\
& \text { MUT-EGFR 3' UTR }
\end{aligned}
$$

B

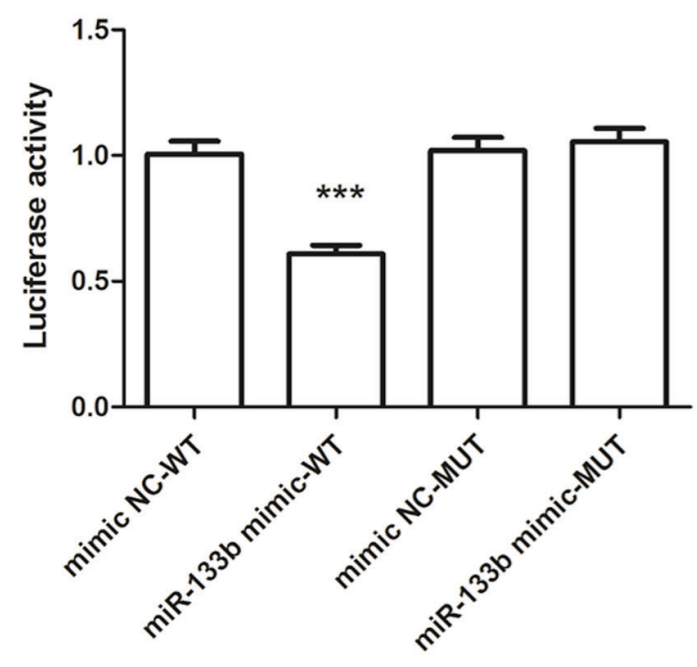

\section{3' -AUCGACCAACUUCCCCUGGUUU-5' \\ 5' -CUCUUUCGAUACCCAGGACCAAG-3' \\ 5' -CUCUUUCGAUACCCACCUGGUUG-3'}

C

Figure 5. miR-133b directly targets the conserved 3'-UTR sequence in EGFR to regulate its expression. (A) Schematic representation of miR-133b and its target sequence (WT or MUT) within the EGFR 3'-UTR of mammals. (B) The luciferase activity of plasmid driven by the WT or MUT EGFR 3'-UTR in SH-SY5Y cells co-transfected with NC or miR-133b mimics. Data are normalized to the mimic NC-WT group. (C) Luciferase activity of WT or MUT EGFR 3'-UTR in the SH-SY5Y cells co-transfected with an NC or miR-133b inhibitor. Data are normalized to the inhibitor NC-WT group. Values are expressed as the mean \pm standard deviation $(\mathrm{n}=5) .{ }^{* * * *} \mathrm{P}<0.001$ vs. the respective NC-WT group. miR, microRNA; NC, negative control; WT, wild-type; MUT, mutant; EGFR, epithelial growth factor receptor; UTR, untranslated region; hsa, Homo sapiens.

relatively simple and effective. Furthermore, with the development of next-generation sequencing technology, the detection of serum miRNAs is becoming increasingly convenient and accurate (30). A vast amount of studies have demonstrated the significant role of aberrant miRNAs in the progression of multiple human diseases (31).

It has been indicated that certain miRNAs are involved in the development, differentiation and synaptic plasticity of neurons; furthermore, their dysregulation may be linked to various diseases, e.g. central nervous system (CNS) diseases, including AD, multiple sclerosis and Huntington's disease (32). To date, a number of miRNAs have been reported to have crucial roles in neurogenesis and neuronal maturation in the CNS (33). Significant differences have been detected regarding miRNA expression levels between AD patients and healthy individuals (34). miRNAs represent a novel class of biomarkers for $\mathrm{AD}$, which are non-invasive and sensitive. miR-133b is general abnormally expressed in various human cancer types, including bladder cancer, breast cancer and glioma (35-37). It has been reported that miR-133b is enriched in the midbrain, which has a marked effect on the differentiation and degeneration of midbrain dopaminergic neurons (12). Furthermore, multiple studies have proved abnormal expression of miR-133b in PD patients. All of this evidence supports the potential role of miR-133b in neurodegenerative disorders.

In the present study, the serum level of miR-133b was significantly downregulated in AD patients, which was consistent with the results of previous studies on PD patients $(14,15)$. The expression of miR-133b was also assessed in the human neuroblastoma cell line SH-SY5Y. The formation of neurofibrillary tangles is regarded to be one of the important pathological characteristics of $\mathrm{AD}$, which may be caused by the accumulation of A $\beta 25-35$ (38). Thus, a cell model of $\mathrm{AD}$ was established by treating SH-SY5Y cells with A $\beta 25-35$, and the concentration of $40 \mu \mathrm{m} / 1$ has been proved to be most efficient (28). The present results demonstrated a significant downregulation of miR-133b in SH-SY5Y cells treated with Aß25-35. Collectively, the present results suggested that miR-133b may be a potential diagnostic biomarker for AD. MMSE is a commonly used screening tool for providing an overall assessment of cognitive impairment in the clinic, and the MMSE score has important value in the diagnosis of mild cognitive impairment that may develop into AD (39). In the present study, the serum levels of miR-133b were proved to be positively correlated with the MMSE scores of AD patients, indicating a significant correlation of miR-133b with the severity of AD and serum miR-133b may therefore be a useful clinical tool for predicting AD occurrence. In order to assess the diagnostic sensitivity and specificity of serum miR-133b for AD, ROC curve analysis was performed. The results suggested that miR-133b is a sensitive biomarker for differentiating AD patients from healthy individuals.

miR-133b is aberrantly expressed in various cancer types, and it may be involved in tumor development via 
regulation of tumor cell proliferation and apoptosis $(40,41)$. The functional role of miR-133b in AD was also explored in a cell model of AD. The A $\beta 25-35$-treated SH-SY5Y cells were transfected with miR-133b mimics, miR-133b inhibitor, mimics NC or inhibitor NC. It was observed that overexpression of miR-133b significantly attenuated the A $325-35$-induced inhibition of cell viability. Furthermore, flow cytometric analysis demonstrated that upregulation of miR-133b prevented A $\beta 25-35$-induced cell apoptosis. All of these results indicated that miR-133b may be involved in the impairment of SH-SY5Y cells treated with A $325-35$. Taken together, it was concluded that miR-133b may have a neuroprotective role in AD.

An important molecular interaction was determined between miR-133b and EGFR in the present study. The TargetScan tool predicted EGFR as a direct target gene of miR-133b. The 3'-UTR of EGFR mRNA was identified to contain a miR-133b seven-nucleotide seed match at position 50-56. The luciferase reporter assay further confirmed that miR-133b targeted the 3'-UTR of EGFR, which was consistent with the bioinformatics prediction.

EGFR, a member of the ErbB family, is located on chromosome 7p12.1-12.3. It has been reported to be involved in cell survival, differentiation, proliferation, migration and repair of cell damage $(42,43)$. Previous studies suggested that EGFR is able to activate several signaling pathways, particularly the mitogen-activated protein kinase/ERK and PI3K/AKT pathways (44), which have been noted to have crucial roles in neuronal growth, survival and plasticity (45). Furthermore, the important role of these two major signaling pathways in AD has been widely demonstrated $(46,47)$. In addition, a key study has indicated that miR-133b inhibits ESCC cells by targeting EGFR (21). All evidence suggests that miR-133b may be involved in the development of AD via targeting EGFR. However, the exact mechanisms require to be fully elucidated in further studies.

In conclusion, the results of the present study suggested that miR-133b may serve as a novel diagnostic biomarker for $\mathrm{AD}$, and it may exert its neuroprotective role in $\mathrm{AD}$ and targets EGFR.

\section{Acknowledgements}

Not applicable.

\section{Funding}

No funding was received.

\section{Availability of data and materials}

All data generated or analyzed during the present study are included in this published article.

\section{Authors' contributions}

YY and QY conceived and designed the experiment. QY and QZ performed all of the experiments. YY, QY and QZ wrote and revised the manuscript. All authors read and approved the final manuscript.

\section{Ethics approval and consent to participate}

The present study was designed under the review and approval of the Ethics Committee of Dongying People's Hospital. Written informed consent was collected from each participant.

\section{Patient consent for publication}

Not applicable.

\section{Competing interests}

The authors declare that they have no competing interests.

\section{References}

1. Balin BJ and Hudson AP: Etiology and pathogenesis of late-onset Alzheimer's disease. Curr Allergy Asthma Rep 14: 417, 2014

2. Gu QF, Yu JZ, Wu H, Li YH, Liu CY, Feng L, Zhang GX, Xiao BG and Ma CG: Therapeutic effect of Rho kinase inhibitor FSD-C10 in a mouse model of Alzheimer's disease. Exp Ther Med 16: 3929-3938, 2018

3. Alzheimer's Association National Plan Milestone Workgroup, Fargo KN, Aisen P, Albert M, Au R, Corrada MM, DeKosky S, Drachman D, Fillit H, Gitlin L, et al: 2014 report on the milestones for the US national plan to address Alzheimer's disease. Alzheimers Dement 10 (5 Suppl): S430-S452, 2014.

4. Mayeux R and Stern Y: Epidemiology of Alzheimer disease. Cold Spring Harb Perspect Med 2: pii: a006239, 2012.

5. Tan MS, Yu JT and Tan L: Bridging integrator 1 (BIN1): Form, function, and Alzheimer's disease. Trends Mol Med 19: 594-603, 2013.

6. Bekris LM, Yu CE, Bird TD and Tsuang DW: Genetics of Alzheimer disease. J Geriatr Psychiatry Neurol 23: 213-227, 2010.

7. Paul S: Integration of miRNA and mRNA expression data for understanding etiology of gynecologic cancers. Methods Mol Biol 1912: 323-338, 2019.

8. Huang X, Liang M, Dittmar R and Wang L: Extracellular microRNAs in urologic malignancies: chances and challenges. Int J Mol Sci 14: 14785-14799, 2013.

9. Kumar S, Vijayan M and Reddy PH: MicroRNA-455-3p as a potential peripheral biomarker for Alzheimer's disease. Hum Mol Genet 26: 3808-3822, 2017.

10. Hong $\mathrm{H}, \mathrm{Li} \mathrm{Y}$ and $\mathrm{Su} \mathrm{B}$ : Identification of circulating miR-125b as a potential biomarker of Alzheimer's disease in APP/PS1 transgenic mouse. J Alzheimers Dis 59: 1449-1458, 2017.

11. Tao J, Wu D, Xu B, Qian W, Li P, Lu Q, Yin C and Zhang W: microRNA-133 inhibits cell proliferation, migration and invasion in prostate cancer cells by targeting the epidermal growth factor receptor. Oncol Rep 27: 1967-1975, 2012.

12. Kim J, Inoue K, Ishii J, Vanti WB, Voronov SV, Murchison E, Hannon G and Abeliovich A: A MicroRNA feedback circuit in midbrain dopamine neurons. Science 317: 1220-1224, 2007.

13. Goedert M: NEURODEGENERATION. Alzheimer's and Parkinson's diseases: The prion concept in relation to assembled $\mathrm{A} \beta$, tau, and $\alpha$-synuclein. Science 349: 1255555, 2015.

14. Zhao N, Jin L, Fei G, Zheng Z and Zhong C: Serum microRNA-133b is associated with low ceruloplasmin levels in Parkinson's disease. Parkinsonism Relat Disord 20: 1177-1180, 2014.

15. Zhang X, Yang R, Hu BL, Lu P, Zhou LL, He ZY, Wu HM and Zhu JH: Reduced circulating levels of miR-433 and miR-133b are potential biomarkers for Parkinson's disease. Front Cell Neurosci 11: 170, 2017.

16. Niu M, Xu R, Wang J, Hou B and Xie A: MiR-133b ameliorates axon degeneration induced by $\mathrm{MPP}(+)$ via targeting RhoA. Neuroscience 325: 39-49, 2016.

17. Igawa S, Ryuge S, Ichinoe M, Nakashima H, Otani S, Nakahara $Y$, Fukui T, Sasaki J, Kubota M, Katagiri M, et al: Impact of EGFR-tyrosine kinase inhibitors on postoperative recurrent non-small-cell lung cancer harboring EGFR mutations. Oncol Res Treat 40: 7-13, 2017. 
18. Tanaka T, Kaida T, Yokoi K, Ishii S, Nishizawa N, Kawamata H, Katoh $\mathrm{H}$, Sato T, Nakamura T, Watanabe M and Yamashita $\mathrm{K}$ Critical relevance of genomic gains of PRL-3/EGFR/c-myc pathway genes in liver metastasis of colorectal cancer. Oncol Lett 17: 1257-1266, 2019.

19. Guo N, Zhao Y, Zhang W, Li S and Yu J: MicroRNA-133a downregulated EGFR expression in human non-small cell lung cancer cells via AKT/ERK signaling. Oncol Lett 16: 6045-6050, 2018.

20. Bruban J, Voloudakis G, Huang Q, Kajiwara Y, Al Rahim M, Yoon Y, Shioi J, Gama Sosa MA, Shao Z, Georgakopoulos A and Robakis NK: Presenilin 1 is necessary for neuronal, but not glial, EGFR expression and neuroprotection via $\gamma$-secretase-independent transcriptional mechanisms. FASEB J 29: 3702-3712, 2015.

21. Zeng W, Zhu JF, Liu JY, Li YL, Dong X, Huang H and Shan L: miR-133b inhibits cell proliferation, migration and invasion of esophageal squamous cell carcinoma by targeting EGFR. Biomed Pharmacother 111: 476-484, 2018.

22. Lugli G, Cohen AM, Bennett DA, Shah RC, Fields CJ, Hernandez AG and Smalheiser NR: Plasma exosomal miRNAs in persons with and without Alzheimer disease: Altered expression and prospects for biomarkers. PLoS One 10: e0139233, 2015.

23. Whitworth JA; World Health Organization, International Society of Hypertension Writing Group: 2003 World Health Organization (WHO)/International Society of Hypertension (ISH) statement on management of hypertension. J Hypertens 21: 1983-1992, 2003.

24. Puavilai G, Chanprasertyotin S and Sriphrapradaeng A Diagnostic criteria for diabetes mellitus and other categories of glucose intolerance: 1997 criteria by the expert committee on the diagnosis and classification of diabetes mellitus (ADA), 1998 WHO consultation criteria, and 1985 WHO criteria. World Health Organization. Diabetes Res Clin Pract 44: 21-26, 1999.

25. Zhang B, Qiu Q, Yin L, Yao Y, Wang C and Wu X: Measurement of retinal function with flash-electroretinography in Chinese patients with hyperlipidemia. Graefes Arch Clin Exp Ophthalmol 252: 1385-1392, 2014

26. Wang X, Zhao X, Li L, Yao H, Jiang Y and Zhang J: Effects of Combination of ezetimibe and rosuvastatin on coronary artery plaque in patients with coronary heart disease. Heart Lung Circ 25: 459-465, 2016.

27. Li H, Jia J and Yang Z: Mini-mental state examination in elderly chinese: A population-based normative study. J Alzheimers Dis 53: 487-496, 2016

28. Hu L, Zhang R, Yuan Q, Gao Y, Yang MQ, Zhang C, Huang J, Sun Y, Yang W, Yang JY, et al: The emerging role of microRNA-4487/6845-3p in Alzheimer's disease pathologies is induced by A $\beta 25-35$ triggered in SH-SY5Y cell. BMC Syst Biol 12 (Suppl 7): S119, 2018.

29. Livak KJ and Schmittgen TD: Analysis of relative gene expression data using real-time quantitative PCR and the 2(-Delta Delta C(T)) method. Methods 25: 402-408, 2001.

30. Amodio N, Stamato MA, Gullà AM, Morelli E, Romeo E, Raimondi L, Pitari MR, Ferrandino I, Misso G, Caraglia M, et al: Therapeutic targeting of miR-29b/HDAC4 epigenetic loop in multiple myeloma. Mol Cancer Ther 15: 1364-1375, 2016.

31. Tian Y, Yan M, Zheng J, Li R, Lin J, Xu A, Liang Y, Zheng R and Yuan Y: miR-483-5p decreases the radiosensitivity of nasopharyngeal carcinoma cells by targeting DAPK1. Lab Invest 99 602-611, 2019.
32. Maniati MS, Maniati M, Yousefi T, Ahmadi-Ahangar A and Tehrani SS: New insights into the role of microRNAs and long noncoding RNAs in most common neurodegenerative diseases. J Cell Biochem 120: 8908-8918, 2019.

33. Díaz NF, Cruz-Reséndiz MS, Flores-Herrera H, García-López G and Molina-Hernández A: MicroRNAs in central nervous system development. Rev Neurosci 25: 675-686, 2014.

34. Maldonado-Lasuncion I, Atienza M, Sanchez-Espinosa MP and Cantero JL: Aging-related changes in cognition and cortical integrity are associated with serum expression of candidate MicroRNAs for Alzheimer disease. Cereb Cortex, Dec 22, 2018 (Epub ahead of print).

35. Shi Z, Kadeer A, Wang M, Wen B, Li M, Huang J, Gao Y, Liu E, Liu D, Jia D and Liang C: The deregulation of miR-133b is associated with poor prognosis in bladder cancer. Pathol Res Pract 215: 354-357, 2019

36. Wang QY, Zhou CX, Zhan MN, Tang J, Wang CL, Ma CN, He M, Chen GQ, He JR and Zhao Q: MiR-133b targets Sox9 to control pathogenesis and metastasis of breast cancer. Cell Death Dis 9: $752,2018$.

37. Zhang Q, Fan X, Xu B, Pang Q and Teng L: miR-133b acts as a tumor suppressor and negatively regulates EMP2 in glioma. Neoplasma 65: 494-504, 2018.

38. Thaker AA, Weinberg BD, Dillon WP, Hess CP, Cabral HJ, Fleischman DA, Leurgans SE, Bennett DA, Hyman BT, Albert MS, et al: Entorhinal cortex: Antemortem cortical thickness and postmortem neurofibrillary tangles and amyloid pathology. AJNR Am J Neuroradiol 38: 961-965, 2017.

39. Arevalo-Rodriguez I, Smailagic N, Roqué I Figuls M, Ciapponi A, Sanchez-Perez E, Giannakou A, Pedraza OL, Bonfill Cosp X and Cullum S: Mini-mental state examination (MMSE) for the detection of Alzheimer's disease and other dementias in people with mild cognitive impairment (MCI). Cochrane Database Syst Rev: CD010783, 2015.

40. Li H, Xiang Z, Liu Y, Xu B and Tang J: MicroRNA-133b inhibits proliferation, cellular migration, and invasion via targeting LASP1 in hepatocarcinoma cells. Oncol Res 25: 1269-1282, 2017.

41. Zhen Y, Liu J, Huang Y, Wang Y, Li W and Wu J: miR-133b inhibits cell growth, migration, and invasion by targeting MMP9 in non-small cell lung cancer. Oncol Res 25: 1109-1116, 2017.

42. Appert-Collin A, Hubert P, Crémel G and Bennasroune A: Role of ErbB receptors in cancer cell migration and invasion. Front Pharmacol 6: 283, 2015.

43. Kim SB, Ly P, Kaisani A, Zhang L, Wright WE and Shay JW: Mitigation of radiation-induced damage by targeting EGFR in noncancerous human epithelial cells. Radiat Res 180: 259-267, 2013.

44. Yarden Y and Sliwkowski MX: Untangling the ErbB signalling network. Nat Rev Mol Cell Biol 2: 127-137, 2001.

45. Guillot F, Kemppainen S, Lavasseur G, Miettinen PO, Laroche S, Tanila H and Davis S: Brain-specific basal and novelty-induced alternations in PI3K-Akt and MAPK/ERK signaling in a middle-aged A $\beta P P / P S 1$ mouse model of Alzheimer's disease. J Alzheimers Dis 51: 1157-1173, 2016

46. Feng MG, Liu CF, Chen L, Feng WB, Liu M, Hai H and Lu JM: MiR-21 attenuates apoptosis-triggered by amyloid- $\beta$ via modulating PDCD4/PI3K/AKT/GSK-3 $\beta$ pathway in SH-SY5Y cells. Biomed Pharmacother 101: 1003-1007, 2018.

47. Zang G, Fang L, Chen L and Wang C: Ameliorative effect of nicergoline on cognitive function through the PI3K/AKT signaling pathway in mouse models of Alzheimer's disease. Mol Med Rep 17: 7293-7300, 2018. 